\title{
FACTORES SOCIODEMOGRÁFICOS Y CLÍNICOS ASOCIADOS A LA RECEPCIÓN DE CUIDADO INFORMAL EN PACIENTES CON NEOPLASIA HEMATOLÓGICA: ESTUDIO BASADO EN LAS DIFERENTES ETAPAS DEL TRATAMIENTO (*)
}

\author{
Marta Ortega-Ortega (1), Roberto Montero-Granados (1) y Antonio Romero-Aguilar (2).
}

(1) Departamento de Economía Aplicada. Facultad de Ciencias Económicas y Empresariales. Universidad de Granada

(2) Servicio de Hematología. Hospital Universitario Virgen de las Nieves. Granada.

(*) "Incentivos para la formación de personal docente e investigador predoctoral en las Universidades Públicas de Andalucía, en áreas de conocimiento consideradas deficitarias por necesidades docentes. Convocatoria 2009" financiada por la Consejería de Innovación, Ciencia y Empresa de la Junta de Andalucía y Fondos Europeos de Desarrollo Regional.

Los autores declaran que no existen conflictos de interés.

\section{RESUMEN}

Fundamentos: Los factores relacionados con la recepción de cuidado informal (CI) en pacientes oncológicos es una cuestión poco estudiada. El objetivo fue analizar los factores sociodemográficos y clínicos asociados a la recepción de cuidado informal en pacientes con neoplasia hematológica a lo largo de las diferentes etapas del tratamiento.

Métodos: 139 pacientes diagnosticados de neoplasia hematológica que recibieron un trasplante de células madre durante el período 2006-2011 en dos centros sanitarios españoles completaron la encuesta elaborada para el estudio. Se estimó un modelo de regresión logística binaria para cada una de las cuatro etapas de tratamiento: pretrasplante, primer año, segundo y tercer año, cuarto al sexto año postrasplante. La variable dependiente fue recibir o no cuidado informal.

Resultados: Pacientes diagnosticados de leucemia aguda presentaron mayor probabilidad de recibir $\mathrm{CI}$ durante la etapa pretrasplante $(\mathrm{OR}=6,394)$ y durante el segundo y tercer año postrasplante $(\mathrm{OR}=42,212)$. A largo plazo $\left(4^{\circ}-6^{\circ}\right.$ año) los pacientes con mieloma múltiple fueron los que requirieron mayor cuidado $(\mathrm{OR}=15,977)$. El estado de salud fue estadísticamente significativo en la mayoría de las etapas. Ser hombre $(\mathrm{OR}=0,263)$, tener pareja $(\mathrm{OR}=0,137)$ y estar empleado $(\mathrm{OR}=0,110)$ se asociaron a una menor probabilidad de recibir CI a largo plazo.

Conclusiones: El CI está presente en más del 75\% de los pacientes con neoplasia hematológica durante la etapa pretrasplante y primer año postrasplante. El diagnóstico y estado de salud son decisivos en la probabilidad de recibirlo, pero no el tipo de trasplante. Los factores sociodemográficos cobran protagonismo a largo plazo.

Palabras clave: Atención al paciente. Cuidadores. Neoplasia. Trasplante. Leucemia. Linfoma. Mieloma Múltiple. España.

Correspondencia

Marta Ortega Ortega

Departamento de Economía Aplicada

Facultad de Ciencias Económicas y Empresariales

Universidad de Granada

Campus de la Cartuja s/n, C.P. 18071, Granada

Correo electrónico: mortega2@ugr.es

\section{ABSTRACT \\ Sociodemographic and Clinical Factors Associated with Informal Care in Hematologic Malignancy Patients: a Study Based on Different Phases of the Treatment, Spain}

Background: There is little information on factors related to use of to informal care in cancer patients. Our objective is to study sociodemographic and clinical factors associated with use of informal care in patients with hematologic malignancy and analyze how these changes throughout different phases of the treatment.

Methods: 139 patients diagnosed with hematologic malignancy who received an haematopoietic stem cell transplantation between 2006-2011 in two Spanish hospitals completed the developed postal questionnaire. A binary logistic regression model was used to analyse the factors associated with use of informal care each of four phases of the treatment (pretransplant, first year, second and third year, and from the fouth to sixth year postransplant). Dependent variable was receive vs. not receive informal care.

Results: Patients diagnosed with acute leukemia had higher probability of receiving informal care during pretransplant period $(\mathrm{OR}=6.394)$ and during the second and third year postransplantation $(\mathrm{OR}=42.212)$. In the longterm (4-6 years), multiple myeloma patients were the ones who required more informal care $(\mathrm{OR}=15.977)$. Health status was statistically significant during all phases. Being male $(\mathrm{OR}=0.263)$, having partner $(\mathrm{OR}=0.137)$ and being employed $(\mathrm{OR}=0.110)$ were associated with lower likelihood of receiving informal care in the long-term.

Conclusions: Over $75 \%$ of patients diagnosed with hematologic malignancy received informal care during pretrasplant and first year postransplant. Type of diagnosis and health status are decisive factors in the probability of receiving informal care at all phases, while the type of transplantation is not. Sociodemografic factors are relevant in the long-term.

Keywords: Patient care. Caregivers. Neoplasm. Transplantation. Leukemia. Lymphoma. Multiple Mieloma. Spain.

DOI: 


\section{INTRODUCCIÓN}

El conjunto de neoplasias hematológicas conforma un grupo de enfermedades malignas que proviene de la expansión clonal de células hematopoyéticas. Enfermedades como la leucemia, el linfoma y el mieloma múltiple forman parte de los veintiocho tipos de cáncer más frecuentes de 184 países, según señala la Agencia Internacional para la Investigación del Cáncer ${ }^{1}$. El tratamiento asociado a las neoplasias hematológicas supone la administración de los correspondientes ciclos de quimioterapia y/o radioterapia con una duración media aproximada de seis meses que, generalmente en las enfermedades citadas, desemboca en un trasplante de progenitores hematopoyéticos (TPH), más conocido como trasplante de médula ósea, permitiendo incrementar la supervivencia a largo plazo $^{2-4}$. Los avances clínicos y tecnológicos han ampliado la posibilidad de obtención de un donante compatible no sólo de hermanos antígeno leucocitario humano (HLA) idénticos (trasplante alogénico emparentado) sino también de cualquier donante no familiar (trasplante alogénico no emparentado) o, incluso, permiten que el paciente sea su propio donante (trasplante autólogo) $)^{2}$.

La agresividad tanto del tratamiento quimioterápico como la del trasplante conlleva la aparición de múltiples efectos secundarios tales como vómitos, mucositis, fatiga, infecciones, malestar, dolor, etcétera, así como problemas de ansiedad y depresión ${ }^{2,5,6}$. Estos síntomas coartan de manera importante la actividad diaria, requiriéndose en la mayoría de los casos apoyo y atención de un cuidador. Según la Organización Mundial de la Salud se entiende por cuidador informal la persona del entorno del enfermo (familiar o amigo) que asume voluntariamente su cuidado sin tener formación específica $\mathrm{y}$ sin recibir, por lo general, contraprestación económica alguna ${ }^{7}$. En este sentido, cabe señalar el papel fundamental que el cuidador informal ejerce en el cuidado del enfermo ${ }^{8-10}$, aportando apoyo emocional, financiero, cuidado personal y del hogar, ayuda en el transporte o supervisión de la medicación ${ }^{11,12}$.

En el caso específico del cáncer, se ha puesto de manifiesto que estos cuidados generan sobre el cuidador informal un mayor estrés en comparación con los cuidados informales prestados a pacientes diagnosticados de otras enfermedades ${ }^{13}$. Cabe destacar, por tanto, la sobrecarga emocional y funcional así como las repercusiones en la salud de cuidadores informales de pacientes oncológicos ${ }^{14,15}$.

Es por ello que diversos trabajos han estimado el tiempo dedicado a cuidados informales en pacientes oncológicos, su valoración monetaria y los posibles factores $\operatorname{asociados}^{16,17}$. En este sentido, diagnósticos como el cáncer colorrectal, de pulmón o próstata han recibido especial atención ${ }^{18-21}$ pero hasta el momento ninguno se ha centrado en el cáncer hematológico.

Sin embargo, previamente al estudio del tiempo y valor monetario asociado a los cuidados informales en pacientes con diferentes neoplasias, es interesante examinar qué factores están relacionados con la necesidad de recibir cuidado informal para poder identificar a la población más vulnerable. Hasta nuestro conocimiento, sólo un estudio enfocado en pacientes oncológicos abordó esta cuestión, señalando que factores como una mayor comorbilidad, encontrarse en la fase terminal de la enfermedad o tener metástasis, están relacionados fuertemente con la necesidad de recibir apoyo en el cuidado personal, transporte y actividades instrumentales en pacientes oncológicos (preparación de la comida, compras, tareas domésticas ${ }^{22}$. Asimismo, las mujeres tienen mayor probabilidad que los hombres de necesitar cuidados en la realización de tareas instrumentales y en actividades relacionadas con el transporte, y los individuos mayores de 65 años y de bajos ingresos revelan mayor necesidad de cuidado personal. Por otro lado, 
Hayman et al. ${ }^{17}$ señalan que la probabilidad de recibir cuidado informal es significativamente mayor en aquellos individuos que recibieron tratamiento oncológico durante el año anterior en comparación con los que no lo han recibido o no han sufrido cáncer. En cualquier caso, como señalan Yabroff et al. ${ }^{16}$ la demanda de cuidados informales varía de manera considerable en base al tipo de diagnóstico y fase del tratamiento en que se encuentre el paciente. En este sentido, hasta nuestro conocimiento, ningún estudio nacional ni internacional ofrece información acerca de la recepción de cuidado informal en pacientes oncohematológicos. Sin embargo, su estudio es relevante fundamentalmente por dos razones: la primera se refiere a la elevada incidencia de nuevos casos de cáncer hematológico y de trasplantes de médula ósea previstos para los próximos años tanto en España como en la región europea ${ }^{3,24}$. Y la segunda es que se ha demostrado que los cuidadores informales de estos pacientes son de los más vulnerables, llegando a experimentar cargas emocionales y costes sociales similares a los de los propios pacientes ${ }^{23}$. Por ello es necesario aportar luz al conocimiento que permita identificar necesidades sociales entre los pacientes oncohematológicos y sus cuidadores para anticipar posibles políticas públicas.

El objetivo del presente trabajo es estudiar los factores sociodemográficos y clínicos asociados a la recepción de cuidado informal en pacientes con neoplasia hematológica y analizar su evolución a lo largo de las diferentes etapas del tratamiento.

\section{SUJETOS Y MÉTODOS}

Diseño del estudio. Dada la ausencia de datos públicos disponibles en España, se diseñó un cuestionario descriptivo para la obtención de información sociodemográfica y clínica de los pacientes. La muestra seleccionada incluyó pacientes diagnosticados de leucemia aguda, linfoma (Hodgkin y no Hodgkin), mieloma múltiple y otras neoplasias menos comunes (síndrome mielodisplásico, leucemia mieloide crónica o leucemia linfoide crónica, entre otros) que habían recibido un trasplante de progenitores hematopoyéticos durante el período 2006 al 2011 en los Hospitales Universitarios Virgen de las Nieves de Granada y Virgen del Rocío de Sevilla (España) que habían sobrevivido a la enfermedad. Se obtuvo la aprobación del Comité Ético de Investigación y del Servicio de Hematología de ambos centros sanitarios.

El cuestionario incluyó una selección de variables sociodemográficas y clínicas tanto estáticas como dinámicas (las que varían en función de la etapa del tratamiento en que se encuentre el paciente). Se diferenciaron cuatro etapas con el objetivo de analizar la evolución temporal en la recepción de cuidado informal.

La etapa 1 se entiende como el período que comprende los ciclos de quimioterapia previos al trasplante. La etapa 2 (corto plazo) fue la correspondiente al transcurso del primer año desde que al paciente se le realizó el trasplante de progenitores hematopoyéticos. Este primer año es de vital importancia dado que pueden aparecer importantes comorbilidades asociadas al trasplante, infecciones y determinadas complicaciones, así como el desarrollo de enfermedad de injerto contra huésped (en el caso del trasplante alogénico) que en muchos casos puede poner en riesgo la vida del paciente. La etapa 3 o medio plazo incluyó el segundo y tercer año posteriores a la realización del trasplante, caracterizada por una situación clínica con cierto nivel de estabilidad, esto es, menor posibilidad de que se produzcan situaciones extremas como el fallecimiento. Por último, la etapa 4 (largo plazo) engloba los años cuarto, quinto y sexto ulteriores al trasplante. La encuesta se realizó cuando los pacientes se encontraban en las etapas 3 o 4, por lo que los sujetos respondieron a las cuestiones relativas a las etapas anteriores basándose en recuerdos. 
Se contactó por teléfono con todos los pacientes diagnosticados y trasplantados en ambos hospitales. Los pacientes que respondieron a la primera o segunda llamada fueron informados e invitados a participar en el estudio. El cuestionario les fue enviado por correo postal junto con una hoja de información, el consentimiento informado, un sobre y un sello para la devolución de la encuesta. La información de contacto se facilitó en caso de duda durante la cumplimentación de la encuesta ${ }^{25}$. La selección de los pacientes comenzó en enero de 2012 y finalizó en diciembre de 2013. El cuestionario fue enviado a 230 pacientes y se devolvieron cumplimentados un total de 139 (tasa de respuesta $60,43 \%$ ).

Análisis estadístico. Se realizó un análisis descriptivo de la muestra objeto de estudio. Posteriormente, de acuerdo a los objetivos, se estimaron cuatro modelos de regresión logística binaria debido al carácter de la variable dependiente binaria y la potencia interpretativa del modelo ${ }^{26}$. En concreto, se estimaron cuatro modelos de acuerdo a la etapa en los que la variable explicada fue la recepción de cuidado informal por parte del paciente oncohematológico durante el periodo correspondiente $\left(\mathrm{y}_{\mathrm{i}}=1\right.$ si recibió cuidado informal; $y_{i}=0$, si no lo recibió, con $\mathrm{i}=1, . ., \mathrm{n}$, siendo $n$ el tamaño de la muestra). Estos modelos permitieron valorar las características personales del individuo cuyos parámetros resultaron estadísticamente significativos y que, por tanto, se asociaron a la recepción de cuidado informal. En este sentido, los odds-ratio midieron la asociación entre la variable dependiente (recibir o no cuidados informales) y cada una de las variables independientes (características sociodemográficas y clínicas) ajustando por el resto de ellas.

Las características sociodemográficas estudiadas fueron el sexo, la edad del paciente en el momento del trasplante (para el análisis fue centralizada en la mediana), el estado civil (con pareja: casado/con pareja, sin pa- reja: soltero/viudo/separado/divorciado), el nivel educativo (nivel bajo: sin estudios/estudios primarios; nivel medio: estudios secundarios/ bachiller/formación profesional de grado medio; nivel alto: estudios universitarios/formación profesional de grado superior), que tras haberse estudiado su condición de linealidad (sigue una distribución de probabilidad lineal y es monótonamente decreciente) fue centrada en el nivel educativo medio, situación laboral (empleado: por cuenta ajena/autónomo; no empleado: ama de casa/estudiante/pensionista/desempleado) y cuidado formal (recibe; no recibe) entendido como el cuidado dedicado a la atención personal y del hogar por parte de un profesional ${ }^{27}$.

Por otro lado, se tuvieron en cuenta características clínicas del individuo, entre las que se incluyeron el estado subjetivo de salud mostrado en una escala de 1 a 5 (1: estado de salud muy malo; 2 : estado de salud malo; 3: estado de salud regular; 4: estado de salud bueno; 5: estado de salud muy bueno). Tras estudiarse la condición de linealidad de dicha variable se introdujo en el modelo como variable continua centrada en el estado de salud regular, siendo por tanto este el valor de referencia de dicha variable en el modelo. El tipo de diagnóstico fue también una característica clínica incluida en los modelos (leucemia aguda/linfoma (de Hodgkin y de No Hodgkin)/mieloma múltiple/otros). Asimismo, cabe señalar que en las fases posteriores a la etapa 1 fue incluida como adicional la variable que recogía el tipo de trasplante recibido en función del donante de células madre (trasplante autólogo/trasplante alogénico). La variable situación laboral no se pudo incluir en el modelo correspondiente a la etapa 4 debido a la falta de variabilidad en la muestra.

Adicionalmente se testó la relación significativa en la recepción de cuidado informal entre las diferentes etapas mediante un contraste no paramétrico para muestras relacionadas con el test $\mathrm{Q}$ de $\mathrm{Cochran}^{28}$, consistente 
en un test $\chi^{2}$ específico para variables dicotómicas. Se utilizó el programa estadístico STATA 12.0 (StataCorp LP, College Station, TX) para la realización de los análisis descritos.

\section{RESULTADOS}

Eran varones 75 (53,96\%), la edad media en el momento del trasplante fue de 46 años (17-67 años), 97 (69,78\%) tenían pareja y 51 $(36,78 \%)$ poseían nivel educativo bajo. 49 habían sido diagnosticados de linfoma (Hodgkin o no Hodgkin) y $90(64,75 \%)$ de los pacientes recibieron un trasplante autólogo (tabla 1).

La tabla 2 describe la evolución de las características sociodemográficas y clínicas por etapas. Se observó una variación de la situación laboral a lo largo del tiempo, en la que 99 $(71,22 \%)$ pacientes se encontraban empleados en la etapa pretrasplante. Sin embargo, a me-

\begin{tabular}{|c|c|c|}
\hline \multicolumn{3}{|c|}{$\begin{array}{c}\text { Tabla 1 } \\
\text { Características sociodemográficas } \\
\text { y clínicas de los pacientes. Variables estáticas }\end{array}$} \\
\hline & n & $\%$ \\
\hline Total & 139 & 100 \\
\hline Edad media en el trasplante & 46,38 & 100 \\
\hline \multicolumn{3}{|l|}{ Sexo } \\
\hline Hombre & 75 & 53,96 \\
\hline Mujer & 64 & 46,04 \\
\hline \multicolumn{3}{|l|}{ Estado Civil } \\
\hline Con Pareja & 97 & 69,78 \\
\hline Sin Pareja & 42 & 30,22 \\
\hline \multicolumn{3}{|l|}{ Nivel educativo } \\
\hline Bajo & 51 & 36,69 \\
\hline Medio & 45 & 32,37 \\
\hline Alto & 43 & 30,94 \\
\hline \multicolumn{3}{|l|}{ Diagnóstico } \\
\hline Leucemia Aguda & 33 & 23,74 \\
\hline Linfoma Hodgkin/no Hodgkin & 49 & 35,25 \\
\hline Mieloma Múltiple & 41 & 29,50 \\
\hline Otro & 16 & 11,51 \\
\hline \multicolumn{3}{|c|}{\begin{tabular}{|l} 
Tipo de trasplante recibido según el donante \\
\end{tabular}} \\
\hline Autólogo & 90 & 64,75 \\
\hline Alogénico: emparentado y no emparentado & 49 & 35,25 \\
\hline
\end{tabular}

dio plazo estas cifras se redujeron drásticamente a $9(12,68 \%)$. A largo plazo se observó una reincorporación al mercado laboral, siendo $22(35,48 \%)$ los que estaban empleados. Por otro lado, se evidenció la importancia de los cuidados informales a lo largo de todas las etapas, fundamentalmente durante el periodo pretrasplante, $115(82,73 \%)$ pacientes, y el primer año postrasplante, recibiéndolo $110(79,14 \%)$ individuos. Los cuidados formales estuvieron presentes en 12 $(8,63 \%)$ durante el pretrasplante. Respecto al estado de salud declarado, $50(35,97 \%)$ lo manifiestaron como "malo" o "muy malo" durante la etapa pretrasplante, reduciéndose al $16,55 \%$ durante el primer año tras el trasplante. A medio y largo plazo, el estado de salud "bueno" predominó en el 43,66\% y $40,32 \%$ de los pacientes, respectivamente (tabla 2).

La estimación de los cuatro modelos de regresión logística binaria aparece recogida en la tabla 3. Como se puede observar, todos los modelos resultaron, a nivel global, estadísticamente significativos. Asimismo, según reveló el porcentaje de ajuste del modelo, todos mostraron un buen ajuste medido a través de las curvas ROC, oscilando entre el $80,07 \%$ (primera etapa) y el $90,95 \%$ (tercera etapa).

En la etapa previa al trasplante, fueron las variables leucemia aguda y estado de salud las que mostraron parámetros estadísticamente significativos. En concreto, individuos diagnosticados de leucemia aguda tuvieron mayor probabilidad de recibir cuidados informales (frente a no recibirlos) respecto a pacientes diagnosticados de linfoma $(\mathrm{OR}=6,394)$. Asimismo, por cada aumento de una unidad en la valoración del estado de salud declarado por el paciente (de 1 a 5) la probabilidad de recibir cuidado informal se redujo a medida que se pasaba de un nivel de estado de salud al siguiente $(\mathrm{OR}=0,138)$. 


\begin{tabular}{|c|c|c|c|c|c|c|c|c|c|}
\hline \multicolumn{10}{|c|}{$\begin{array}{c}\text { Tabla } 2 \\
\text { Evolución de las características sociodemográficas y clínicas de los pacientes } \\
\text { por etapas. Variables dinámicas }\end{array}$} \\
\hline & & \multicolumn{2}{|c|}{$\begin{array}{c}\text { Etapa 1: } \\
\text { pretrasplante }\end{array}$} & \multicolumn{2}{|c|}{$\begin{array}{l}\text { Etapa 2: } \\
1^{\circ} \text { año } \\
\text { postrasplante }\end{array}$} & \multicolumn{2}{|c|}{$\begin{array}{c}\text { Etapa 3: } \\
2^{\circ}-3^{\circ} \text { año } \\
\text { postrasplante }\end{array}$} & \multicolumn{2}{|c|}{$\begin{array}{c}\text { Etapa 4: } \\
4^{\circ}-6^{\circ} \text { año } \\
\text { postrasplante }\end{array}$} \\
\hline & & $\mathrm{n}$ & $\%$ & $\mathrm{n}$ & $\%$ & $\mathrm{n}$ & $\%$ & $\mathrm{n}$ & $\%$ \\
\hline \multicolumn{2}{|c|}{ Total muestra } & 139 & 100,00 & 139 & 100,00 & 71 & 100,00 & 62 & 100,00 \\
\hline \multicolumn{10}{|c|}{ Recepción de cuidados informales } \\
\hline \multirow{2}{*}{$\begin{array}{l}\text { Leucemia } \\
\text { Aguda }\end{array}$} & Sí & 31 & 93,94 & 29 & 87,88 & 9 & 60,00 & 4 & 26,67 \\
\hline & No & 2 & 6,06 & 4 & 12,12 & 6 & 40,00 & 11 & 73,33 \\
\hline \multirow{2}{*}{$\begin{array}{l}\text { L. Hodgkin/ } \\
\text { no Hodgkin }\end{array}$} & Sí & 36 & 73,47 & 35 & 71,43 & 7 & 26,92 & 4 & 18,18 \\
\hline & No & 13 & 26,53 & 14 & 28,57 & 19 & 73,08 & 18 & 81,82 \\
\hline \multirow{2}{*}{$\begin{array}{l}\text { Mieloma } \\
\text { Múltiple }\end{array}$} & Sí & 35 & 85,37 & 33 & 80,49 & 12 & 63,16 & 13 & 65,00 \\
\hline & No & 6 & 14,63 & 8 & 19,51 & 7 & 36,84 & 7 & 35,00 \\
\hline \multirow{2}{*}{ Otro } & Sí & 13 & 81,25 & 13 & 81,25 & 4 & 36,36 & 1 & 20,00 \\
\hline & No & 3 & 18,75 & 3 & 18,75 & 7 & 63,64 & 4 & 80,00 \\
\hline \multirow{2}{*}{ Total } & Sí & 115 & 82,73 & 110 & 79,14 & 32 & 45,07 & 22 & 35,48 \\
\hline & No & 24 & 17,27 & 29 & 20,86 & 39 & 54,93 & 40 & 64,52 \\
\hline \multicolumn{10}{|c|}{ Recepción de cuidados formales } \\
\hline \multirow{2}{*}{$\begin{array}{l}\text { Leucemia } \\
\text { Aguda }\end{array}$} & Sí & 2 & 6,06 & 2 & 6,06 & 0 & 0,00 & 0 & 0,00 \\
\hline & No & 31 & 93,94 & 31 & 93,94 & 15 & 100,00 & 15 & 100,00 \\
\hline \multirow{2}{*}{$\begin{array}{l}\text { L. Hodgkin/ } \\
\text { no Hodgkin }\end{array}$} & Sí & 2 & 4,08 & 2 & 4,08 & 0 & 0,00 & 1 & 4,55 \\
\hline & No & 47 & 95,92 & 47 & 95,92 & 26 & 100,00 & 21 & 95,45 \\
\hline \multirow{2}{*}{$\begin{array}{l}\text { Mieloma } \\
\text { Múltiple }\end{array}$} & Sí & 8 & 19,51 & 6 & 14,63 & 4 & 21,05 & 4 & 20,00 \\
\hline & No & 33 & 80,49 & 35 & 85,37 & 15 & 78,95 & 16 & 80,00 \\
\hline \multirow{2}{*}{ Otro } & Sí & 0 & 0,00 & 1 & 6,25 & 0 & 0,00 & 0 & 0,00 \\
\hline & No & 16 & 100,00 & 15 & 93,75 & 11 & 100,00 & 5 & 100,00 \\
\hline \multirow{2}{*}{ Total } & Sí & 12 & 8,63 & 11 & 7,91 & 4 & 5,63 & 5 & 8,06 \\
\hline & No & 127 & 91,37 & 128 & 92,09 & 67 & 94,37 & 57 & 91,94 \\
\hline \multicolumn{10}{|c|}{ Situación laboral } \\
\hline \multirow{2}{*}{$\begin{array}{l}\text { Leucemia } \\
\text { Aguda }\end{array}$} & Empleado & 23 & 69,70 & 12 & 36,36 & 2 & 13,33 & 5 & 33,33 \\
\hline & No empleado & 10 & 30,30 & 21 & 63,64 & 13 & 86,67 & 10 & 66,67 \\
\hline \multirow{2}{*}{$\begin{array}{l}\text { L. Hodgkin/ } \\
\text { no Hodgkin }\end{array}$} & Empleado & 33 & 67,35 & 16 & 32,65 & 4 & 15,38 & 11 & 50,00 \\
\hline & No empleado & 16 & 32,65 & 33 & 67,35 & 22 & 84,62 & 11 & 50,00 \\
\hline \multirow{2}{*}{$\begin{array}{l}\text { Mieloma } \\
\text { Múltiple }\end{array}$} & Empleado & 29 & 70,73 & 10 & 24,39 & 2 & 10,53 & 3 & 15,00 \\
\hline & No empleado & 12 & 29,27 & 31 & 75,61 & 17 & 89,47 & 17 & 85,00 \\
\hline \multirow{2}{*}{ Otro } & Empleado & 14 & 87,50 & 6 & 37,50 & 1 & 9,09 & 3 & 60,00 \\
\hline & No empleado & 2 & 12,50 & 10 & 62,50 & 10 & 90,91 & 2 & 40,00 \\
\hline \multirow{2}{*}{ Total } & Empleado & 99 & 71,22 & 44 & 31,69 & 9 & 12,68 & 22 & 35,48 \\
\hline & No empleado & 40 & 28,78 & 95 & 68,35 & 62 & 87,32 & 40 & 64,52 \\
\hline
\end{tabular}




\begin{tabular}{|c|c|c|c|c|c|c|c|c|c|}
\hline \multicolumn{10}{|c|}{$\begin{array}{c}\text { Tabla } 2 \\
\begin{array}{c}\text { Evolución de las características sociodemográficas y clínicas de los pacientes por } \\
\text { etapas. Variables dinámicas (continuación) }\end{array}\end{array}$} \\
\hline & & \multicolumn{2}{|c|}{$\begin{array}{c}\text { Etapa 1: } \\
\text { Pretrasplante }\end{array}$} & \multicolumn{2}{|c|}{$\begin{array}{c}\text { Etapa 2: } \\
1^{\circ} \text { año } \\
\text { postrasplante }\end{array}$} & \multicolumn{2}{|c|}{$\begin{array}{c}\text { Etapa 3: } \\
2^{\mathbf{0}}-3^{\mathbf{0}} \\
\text { año postrasplante }\end{array}$} & \multicolumn{2}{|c|}{$\begin{array}{c}\text { Etapa 4: } \\
4^{\circ}-6^{\circ} \text { año } \\
\text { postrasplante }\end{array}$} \\
\hline & & $\mathrm{n}$ & $\%$ & $\mathbf{n}$ & $\%$ & n & $\%$ & n & $\%$ \\
\hline \multicolumn{10}{|c|}{ Estado de salud } \\
\hline \multirow{5}{*}{$\begin{array}{l}\text { Leucemia } \\
\text { Aguda }\end{array}$} & Muy malo & 5 & 15,15 & 0 & 0,00 & 1 & 6,67 & 0 & 0,00 \\
\hline & Malo & 6 & 18,18 & 8 & 24,24 & 1 & 6,67 & 1 & 6,67 \\
\hline & Regular & 15 & 45,45 & 13 & 39,39 & 6 & 40,00 & 2 & 13,33 \\
\hline & Bueno & 6 & 18,18 & 11 & 33,33 & 3 & 20,00 & 8 & 53,33 \\
\hline & Muy bueno & 1 & 3,03 & 1 & 3,03 & 4 & 26,67 & 4 & 26,67 \\
\hline \multirow{5}{*}{$\begin{array}{l}\text { Linfoma } \\
\text { Hodgkin/ } \\
\text { no Hodgkin }\end{array}$} & Muy malo & 5 & 10,20 & 2 & 4,08 & 0 & 0,00 & 0 & 0,00 \\
\hline & Malo & 11 & 22,45 & 5 & 10,20 & 0 & 0,00 & 0 & 0,00 \\
\hline & Regular & 19 & 38,78 & 19 & 38,78 & 10 & 38,46 & 5 & 22,73 \\
\hline & Bueno & 13 & 26,53 & 21 & 42,86 & 14 & 53,85 & 4 & 18,18 \\
\hline & Muy bueno & 1 & 2,04 & 2 & 4,08 & 2 & 7,69 & 13 & 59,09 \\
\hline \multirow{5}{*}{$\begin{array}{l}\text { Mieloma } \\
\text { Múltiple }\end{array}$} & Muy malo & 6 & 14,63 & 2 & 4,88 & 0 & 0,00 & 2 & 10,00 \\
\hline & Malo & 11 & 26,83 & 3 & 7,32 & 1 & 5,26 & 1 & 5,00 \\
\hline & Regular & 15 & 36,59 & 15 & 36,59 & 6 & 31,58 & 5 & 25,00 \\
\hline & Bueno & 9 & 21,95 & 18 & 43,20 & 9 & 47,37 & 10 & 50,00 \\
\hline & Muy bueno & 0 & 0,00 & 3 & 7,32 & 3 & 15,79 & 2 & 10,00 \\
\hline \multirow{5}{*}{ Otro } & Muy malo & 4 & 25,00 & 2 & 12,50 & 0 & 0,00 & 0 & 0,00 \\
\hline & Malo & 2 & 12,5 & 1 & 6,25 & 2 & 18,18 & 0 & 0,00 \\
\hline & Regular & 7 & 43,75 & 9 & 56,25 & 2 & 18,18 & 1 & 20,00 \\
\hline & Bueno & 3 & 18,75 & 4 & 25,00 & 5 & 45,45 & 3 & 60,00 \\
\hline & Muy bueno & 0 & 0,00 & 0 & 0,00 & 2 & 18,18 & 1 & 20,00 \\
\hline \multirow{5}{*}{ Total } & Muy malo & 20 & 14,39 & 6 & 4,32 & 1 & 1,41 & 2 & 3,23 \\
\hline & Malo & 30 & 21,58 & 17 & 12,23 & 4 & 5,63 & 2 & 3,23 \\
\hline & Regular & 56 & 40,29 & 56 & 40,29 & 24 & 33,8 & 13 & 20,97 \\
\hline & Bueno & 31 & 22,30 & 54 & 38,85 & 31 & 43,66 & 25 & 40,32 \\
\hline & Muy bueno & 2 & 1,44 & 6 & 4,32 & 11 & 15,49 & 20 & 32,26 \\
\hline
\end{tabular}

En la etapa transcurrida durante el primer año postrasplante, de nuevo la variable estado de salud resultó tener un parámetro estadísticamente significativo $(\mathrm{OR}=0,311)$ junto al sexo. En este sentido, los hombres presentaron una probabilidad menor de recibir cuidado informal $(\mathrm{OR}=0,406)$ en comparación con las mujeres durante esta fase. Cabe destacar que en este periodo los parámetros asociados al diagnóstico y tipo de trasplante no resultaron estadísticamente significativos.

Durante la etapa 3 la probabilidad de recibir cuidado informal fue mayor en quienes padecían leucemia aguda $(\mathrm{OR}=42,212)$ y mieloma múltiple $(\mathrm{OR}=23,036)$ respecto a quienes tenían linfoma. Asimismo, por cada aumento en una unidad en la valoración del estado de salud manifestado se esperaba que la probabilidad de recibir cuidado informal disminuyera $(\mathrm{OR}=0,100)$.

En la etapa 4 del análisis las variables sociodemográficas adquirieron significación estadística, mostrando parámetros estadísticamente significativos la enfermedad de mieloma múltiple, ser hombre, tener pareja, y estar empleado. De esta manera, la proba- 


\begin{tabular}{|c|c|c|c|c|c|c|c|c|}
\hline \multicolumn{9}{|c|}{$\begin{array}{c}\text { Tabla } 3 \\
\text { Modelos de regresión logística por etapas de los factores asociados } \\
\text { a la recepción de cuidado informal en pacientes oncohematológicos }\end{array}$} \\
\hline & \multicolumn{2}{|c|}{$\begin{array}{c}\text { Etapa } 1 \\
\text { Pretrasplante }\end{array}$} & \multicolumn{2}{|c|}{$\begin{array}{c}\text { Etapa } 2 \\
1^{\circ} \text { año } \\
\text { postrasplante }\end{array}$} & \multicolumn{2}{|c|}{$\begin{array}{c}\text { Etapa } 3 \\
2^{\circ}-3^{\circ} \text { año } \\
\text { postrasplante }\end{array}$} & \multicolumn{2}{|c|}{$\begin{array}{c}\text { Etapa } 4 \\
4^{\circ}-6^{\circ} \text { año } \\
\text { postrasplante }\end{array}$} \\
\hline & $\begin{array}{l}\text { Odds } \\
\text { Ratio }\end{array}$ & $\begin{array}{c}\text { Error } \\
\text { estándar }\end{array}$ & $\begin{array}{l}\text { Odds } \\
\text { Ratio }\end{array}$ & $\begin{array}{c}\text { Error } \\
\text { estándar }\end{array}$ & $\begin{array}{l}\text { Odds } \\
\text { Ratio }\end{array}$ & $\begin{array}{c}\text { Error } \\
\text { estándar }\end{array}$ & $\begin{array}{l}\text { Odds } \\
\text { Ratio }\end{array}$ & $\begin{array}{c}\text { Error } \\
\text { estándar }\end{array}$ \\
\hline \multicolumn{9}{|c|}{ Diagnóstico (ref. linfoma) } \\
\hline Leucemia aguda & $6,394^{\dagger}$ & 5,441 & 2,084 & 1,629 & $42,212^{*}$ & 60,623 & 0,355 & 0,688 \\
\hline Mieloma múltiple & 1,512 & 0,996 & 1,508 & 0,915 & $23,036^{*}$ & 24,480 & $15,977^{\dagger}$ & 19,231 \\
\hline Otros & 1,562 & 1,289 & 0,996 & 0,845 & 3,345 & 4,061 & 0,638 & 1,290 \\
\hline $\begin{array}{l}\text { Estado de salud } \\
\text { (ref. regular) }\end{array}$ & $0,318^{*}$ & 0,105 & $0,311^{*}$ & 0,116 & $0,100^{*}$ & 0,063 & 0,607 & 0,263 \\
\hline \multicolumn{9}{|c|}{ Tipo de trasplante (ref. autólogo) } \\
\hline $\begin{array}{l}\text { Trasplante } \\
\text { alogénico }\end{array}$ & - & - & 1,848 & 1,220 & 0,208 & 0,231 & 6,878 & 12,903 \\
\hline \multicolumn{9}{|l|}{ Sexo (ref. mujer) } \\
\hline Hombre & 0,751 & 0,411 & $0,406^{\ddagger}$ & 0,221 & 1,019 & 0,774 & $0,263^{+}$ & 0,210 \\
\hline $\begin{array}{l}\text { Edad en el } \\
\text { trasplante (ref. } 49 \\
\text { años) }\end{array}$ & 0,995 & 0,022 & 1,009 & 0,219 & 1,011 & 0,041 & 0,999 & 0,037 \\
\hline $\begin{array}{l}\text { Nivel educativo } \\
\text { (ref. medio) }\end{array}$ & 0,849 & 0,294 & 1,064 & 0,352 & 0,742 & 0,367 & 1,081 & 0,506 \\
\hline \multicolumn{9}{|l|}{$\begin{array}{l}\text { Estado Civil } \\
\text { (ref. sin pareja) }\end{array}$} \\
\hline Con pareja & 2,088 & 1,334 & 1,132 & 0,748 & 1,821 & 1,793 & $0,137^{*}$ & 0,161 \\
\hline \multicolumn{9}{|l|}{$\begin{array}{l}\text { Situación laboral } \\
\text { (ref. no empleado) }\end{array}$} \\
\hline Empleado & 0,579 & 0,348 & 0,443 & 0,236 & 3,176 & 3,661 & $0,110^{\dagger}$ & 0,119 \\
\hline \multicolumn{9}{|c|}{ Cuidados formales (ref. no recibe) } \\
\hline $\begin{array}{l}\text { Recibe cuidados } \\
\text { formales }\end{array}$ & 1,837 & 2,278 & 1,088 & 1,295 & 0,122 & 0,203 & - & - \\
\hline Constante & 3,294 & 2,543 & $9,927^{*}$ & 7,549 & 0,630 & 0,703 & 2,731 & 3,020 \\
\hline $\mathbf{N}$ & & 139 & & 139 & & 71 & & 62 \\
\hline $\operatorname{LR} \chi^{2}$ & & $26,04^{*}$ & & $28,74^{*}$ & & $42,13^{*}$ & & $30,82^{*}$ \\
\hline Pseudo $\mathbf{R}^{2}$ & & 0,203 & & 0,201 & & 0,431 & & 0,382 \\
\hline Curva ROC & & $80,07 \%$ & & $80,71 \%$ & & $90,95 \%$ & & $87,79 \%$ \\
\hline
\end{tabular}

bilidad de que un paciente oncohematológico recibiera cuidado informal en esta etapa fue superior si el paciente sufría mieloma múltiple respecto de un paciente diagnosticado de linfoma $(\mathrm{OR}=15,977)$. Del mismo modo, como ocurrió durante el primer año tras el trasplante, el hombre tuvo una probabilidad menor de recibir cuidados informales durante esta etapa respecto a la mujer
$(\mathrm{OR}=0,263)$. Por otro lado, la probabilidad de que un paciente con pareja recibiera cuidados informales fue menor respecto de un paciente que no la tenía $(\mathrm{OR}=0,137)$. Y los pacientes empleados tuvieron una probabilidad menor de recibir cuidados informales en comparación con los que sí estaban empleados durante este período $(\mathrm{OR}=0,110)$. Cabe señalar que el parámetro asociado al 


\begin{tabular}{|l} 
Test Q de Cochran. Relación existente entre las diferentes etapas en la recepción \\
de cuidado informal \\
\hline \\
\hline
\end{tabular}

estado de salud dejó de ser estadísticamente significativo en esta etapa.

Por último, el análisis de la constante determinó que en la etapa 1 la probabilidad de recibir cuidado informal teniendo como individuo de referencia una mujer de 49 años diagnosticada de linfoma, con un estado de salud regular, sin pareja, no empleada y que no recibía cuidados formales fue del $76,71 \%$. Durante la etapa 2, manteniendo constantes las características de nuestro individuo de referencia (al que en esta etapa se le realizó previamente un trasplante autólogo) la probabilidad de recibir cuidado informal fue del $90,85 \%$, durante la etapa 3 del $38,65 \%$ y finalmente en la etapa 4 del 73,19\%.

Los resultados del test de Cochran se detallan en la tabla 4, reflejando que no hubo diferencias estadísticamente significativas al recibir cuidados informales durante la etapa pretrasplante y el primer año tras el trasplante $(\mathrm{p}>0,05)$. Esta igualdad desapareció cuando se comparó la recepción de cuidados informales durante la etapa pretrasplante y el primer año tras el trasplante con las etapas 3 y 4 $(\mathrm{p}<0,000)$.

\section{DISCUSIÓN}

De acuerdo con nuestros resultados se observa una presencia predominante del cuidado informal frente al formal durante todas las etapas analizadas. Este cuidado es especialmente más frecuente en la etapa pretrasplante y durante el primer año tras el trasplante, alargándose en el tiempo para quienes han desarrollado complicaciones y efectos secundarios graves o han sufrido una recaí- da en la enfermedad ${ }^{5,6,29}$. Evidenciamos una relación complementaria entre los cuidados informales y formales, bajo el supuesto de que, dada la nula significatición estadística del parámetro asociado al cuidado formal en nuestros modelos, este puede estar presente en determinados casos para mejorar la situación del cuidador y/o del paciente. Esta relación se manifesta en otros estudios ${ }^{19}$, siendo los cuidados formales cada vez más necesarios dados los cambios sociales en las últimas décadas (incorporación de la mujer al trabajo o envejecimiento de la población $)^{30}$. La complementariedad de ambos cuidados se ha observado además en población con dependencia $^{31}$, población de edad avanzada ${ }^{8} \mathrm{o}$ en sujetos con enfermedades como el Alhzeimer (en el caso de cuidados formales en casa $)^{10}$ en España, siendo mucho más frecuente en otros países europeos ${ }^{32}$. Sin embargo, todos ellos coinciden en destacar la elevada importancia del cuidado informal lo que supone de manera añadida un importante ahorro de recursos económicos familiares y públicos ${ }^{8-11,33}$.

Nuestros resultados indican que en la etapa previa al trasplante son los pacientes diagnosticados de leucemia aguda los que muestran mayor probabilidad de recibir cuidado informal, debido a que su tratamiento quimioterápico es mucho más agresivo que el del resto de diagnósticos ${ }^{2}$, por lo que los efectos secundarios y la necesidad de apoyo y ayuda son mayores. Igualmente, la probabilidad de recibir cuidado informal a medio plazo continúa manteniéndose superior. $\mathrm{Su}$ justificación radica en que la probabilidad de recaída durante el primer y segundo año, es del $52 \%$ tras un trasplante autólogo y del 25\% 
tras un trasplante alogénico ${ }^{34}$, mientras que en el caso del linfoma la probabilidad se reduce hasta una media del $35 \%$ y $34 \%$, respectivamente $^{34}$. A largo plazo, la variable leucemia aguda deja de ser estadísticamente significativa, ya que quienes logran sobrevivir consiguen recuperar su calidad de vida casi completamente volviendo a un nivel satisfactorio de bienestar físico, emocional, psicológico y de actividad diaria ${ }^{6}$.

Tendencia opuesta presenta el diagnóstico de mieloma múltiple siendo el que mayor necesidad de cuidado informal demanda a medio y largo plazo. La justificación estriba en que, a pesar de que su tratamiento actual ha mejorado sustancialmente en los últimos años en relación a la prolongación de la supervivencia global del paciente, con la introducción de altas dosis de quimioterapia combinado con un trasplante de células madre autólogo, la tasa de supervivencia a los cinco años del trasplante permanece pobre, siendo del $35-37 \%$ y la probabilidad de recaída a los tres y cuatro años es del $90 \%{ }^{34}$.

Relevante interés muestran las variables diagnóstico y tipo de trasplante durante la etapa 2, no siendo ninguna de ellas determinante en la recepción de cuidado informal. La justificación puede radicar en que el TPH es uno de los procedimientos clínicos más complejos debido a los efectos secundarios asociados y a la notable reducción de la calidad de vida del paciente, lo que sugiere una implicación por parte de los familiares similar independientemente del diagnóstico y del tipo de trasplante ${ }^{29}$.

El estado de salud se convierte en una variable de valiosa importancia en la recepción de cuidado informal ${ }^{22}$. De este modo, una mejor percepción del estado de salud por parte del paciente redunda en menores necesidades de cuidado informal, tanto en la fase pretrasplante como durante el primer, segundo y tercer año. Por el contrario, a largo plazo, el estado de salud deja de ser estadísticamente significativo. Ello se debe a que la variable situación laboral recoge el efecto del estado de salud de los pacientes, puesto que a largo plazo, aquéllos que tienen buen estado de salud suelen encontrarse trabajando al superar en gran medida la enfermedad y por el contrario no es frecuente que trabajen quienes carecen de buen estado de salud.

Es relevante señalar que los factores sociodemográficos incluidos en todas las etapas no resultan estadísticamente significativos en la recepción de cuidado informal en detrimento de los clínicos. Estos resultados se corroboran con otros estudios en los que factores asociados al cuidador y/o paciente, como la $\operatorname{edad}^{16,19,20}$, sexo ${ }^{16,19}$, raza ${ }^{16}$, estado civil ${ }^{16} \mathrm{o}$ situación laboral ${ }^{20}$ no están relacionados de forma estadísticamente significativa con el tiempo dedicado a los cuidados informales en pacientes oncológicos. Sin embargo, en nuestro estudio, el sexo resulta estadísticamente significativo en las etapas 2 y 4 , reflejándose que las mujeres tienen mayor probabilidad de recibir cuidado informal respecto a los hombres. Esto puede ser debido a que manifiestan mayor necesidad de ayuda en el desarrollo de actividades instrumentales y de transporte que los hombres ${ }^{22}$, en concreto, en etapas tan decisivas como son el primer año tras el trasplante y una posible reaparición de la enfermedad a largo plazo. Asimismo, es destacable que tener pareja, a largo plazo se asocia a una menor probabilidad de recibir cuidado informal. Siguiendo la línea de Rogero $^{8}$, este hecho se puede deber al posible sesgo en la identificación del tipo de cuidado por parte de los individuos con pareja, al no identificar como tal el cuidado informal de su cónyuge, sino que lo consideran como un tipo de ayuda normal, difícilmente distinguible de la que se proveía anteriormente.

Por último, los cuidadores que han prestado su cuidado durante la etapa pretrasplante lo continuarán haciendo durante el primer año tras el trasplante. Sin embargo, no existe dicha continuidad de cuidados a medio y largo plazo $^{17}$.

Una de las limitaciones del presente trabajo se refiere al tamaño de la muestra, principal- 
mente motivado por la falta de información disponible en fuentes oficiales. Asimismo, la selección de pacientes trasplantados puede conllevar un posible sesgo motivado por el objetivo de analizar no sólo la etapa quimioterápica sino también las fases inherentes a un posible tratamiento posterior. En esta línea, para futuras investigaciones se recomienda un mayor tamaño de muestra y pacientes no trasplantados para el contraste de nuestros resultados. Una segunda limitación se refiere a la utilización del método basado en el recuerdo para la respuesta a determinadas preguntas dinámicas formuladas en el cuestionario, debido al posible sesgo inherente a la memoria.

En conclusión, el presente trabajo se configura como un posible punto de partida de una nueva línea de investigación en el área oncohematológica, de futuro interés si se atiende a la elevada incidencia de nuevos casos estimados para el año 2020 en la región europea, donde se estima un aumento de nuevos casos en comparación con el año 2012 del 9,75\% para la leucemia, del 8,51\% para el linfoma y del $11,95 \%$ para el mieloma múltiple ${ }^{24}$.

En base a la información tratada y evocando la construcción del cuarto pilar del Estado de Bienestar en España, con la denominada Ley de Promoción de la Autonomía Personal y Atención a las Personas en situación de Dependencia (LAPAD) ${ }^{36}$, y tras la Resolución publicada en el año $2010^{37}$, queremos hacer mención al punto 8.1, apartado "Aspectos relativos al procedimiento", que especifica que "personas con procesos patológicos graves que requieren tratamientos agresivos que condicionan una situación de dependencia transitoria asociada al propio tratamiento (quimioterapia, radioterapia, otros tratamientos inmunosupresores, inmovilizaciones, tracciones...), el trámite de la solicitud procederá una vez finalizado el tratamiento y haya alcanzado la máxima recuperación funcional". Esta resolución limita la posibilidad de los pacientes onco- lógicos de acogerse a las ayudas ofrecidas por la LAPAD hasta su completa recuperación. Sin embargo, según se manifiesta en el presente estudio, el cuidador informal desempeña un rol fundamental en el cuidado y apoyo a pacientes oncohematológicos, principalmente durante la etapa pretrasplante y el primer año tras el trasplante. Por otro lado, si se añade que los cuidadores informales de pacientes oncológicos presentan mayor estrés en la atención de su familiar en comparación con otras enfermedades ${ }^{13}$, no parece justificado que dichos pacientes deban quedar excluidos de su aplicación. Sería interesante la creación de un posible servicio de ayuda a domicilio eventual con carácter extraordinario, en el propio marco de la LAPAD, con el fin de contribuir a la reducción de la carga de los cuidadores informales durante las etapas 1 y 2 y mejora de la calidad de vida tanto de éstos como de los propios pacientes.

\section{AGRADECIMIENTOS}

Agradecemos la participación de todas las personas que voluntariamente accedieron a participar en el estudio. Asimismo, agradecemos la colaboración prestada por el Hospital Universitario Virgen de las Nieves de Granada (España) y el Hospital Universitario Virgen del Rocío de Sevilla (España) para la realización de la investigación, en especial a los profesionales del Servicio de Hematología de ambos centros, destacando la colaboración del Dr. D. Ildefonso Espigado Tocino. Igualmente queremos manifestar nuestro agradecimiento al profesor D. Juan de Dios Jiménez Aguilera.

\section{BIBLIOGRAFÍA}

1. Las cifras del cáncer en España 2014. Madrid: Sociedad Española de Oncología Médica; 2014.

2. De Linares Fernández S, Contreras Molina C, Fernández Cordero I. Guía informativa trasplante de médula ósea. Granada: Laboratorios Esteve; 2007

3. Tan SS, Uyl de-Groot CA, Huijgens PC, Fibbe WE. Stem cell transplantation in Europe: trends and prospects. Eur J Cancer. 2007;43(16):2359-2365. 
4. Wingard JR, Majhail NS, Brazauskas R, Wang Z, Sobocinski KA, Jacobsohn D, et al. Long-term survival and late deaths after allogeneic hematopoietic cell transplantation. J Clin Oncol. 2011;29(16):2230-2239.

5. Bieri S, Roosnek E, Helg C, Verholen F, Robert D, Chapuis B, et al. Quality of life and social integration after allogeneic hematopoietic SCT. Bone Marrow Transplant. 2008;42(12):819-827.

6. Redaelli A, Stephens JM, Brandt S, Botteman MF, Pashos CL . Short- and long-term effects of acute myeloid leukemia on patient health-related quality of life. Cancer Treat Rev. 2004;30(1):103117.

7. Word Health Organization Lessons for longterm care policy. The cross-cluster initiative on long-term care. (WHO); 2002.

8. Rogero García J. Distribución en España del cuidado formal e informal a las personas de $65 \mathrm{y}$ más años en situación de dependencia. Rev Esp Salud Pública. 2009;83(3):393-405.

9. Aranda-Reneo I, Oliva-Moreno J, VilaplanaPrieto C, Hidalgo-Vega Á, González-Domínguez A. Informal care of patients with schizophrenia. J Ment Health Policy Econ. 2013;16(3):99-108.

10. Peña-Longobardo LM, Oliva-Moreno J. Economic valuation and determinants of informal care to people with Alzheimer's disease. Eur J Health Econ. 2014 May 7. [Epub ahead of print].

11. Oliva Moreno J, Vilaplana C, Osuna Guerrero R. El valor de los cuidados informales prestados a personas en situación de dependencia en España. Instituto de Estudios Fiscales: Pap Trabajo. 2011;(10):7-48.

12. Given BA, Given CW, Kozanchik S. Family support in advanced care. CA Cancer J Clin. 2001;51(4):213-231.

13. Kim Y, Schulz R. Family caregivers' strains: comparative analysis of cancer caregiving with dementia, diabetes, and frail elderly caregiving. J Aging Health. 2008;20(5):483-503.

14. Rodriguez-Rodriguez A, Rihuete-Galve MI. Influencia de la dependencia de los enfermos oncológicos en la sobrecarga de sus cuidadores familiares. Med Paliat. 2011;18(4):135-140.

15. Goren A, Isabelle Gilloteau I, Lees M, daCosta DiBonaventura M. Quantifying the burden of informal caregiving for patients with cancer in Europe. Support Care Cancer. 2014;22(6):16371646.

16. Yabroff KR, Kim Y. Time costs associated with informal caregiving for cancer survivors. Cancer. 2009;115 Suppl 18:4362-4373.
17. Hayman JA, Langa KM, Kabeto MU, Katz SJ, DeMonner SM, Chernew ME, et al. Estimating the cost of informal caregiving for elderly patients with cancer. J Clin Oncol. 2011;19(13):3219-3225.

18. Hanly P, Déilleachair AO, Skally M, O’Leary E, Staines A, Kapur K et al. Time costs associated with informal care for colorectal cancer: an investigation of the impact of alternative valuation methods. Appl Health Econ Health Policy. 2013;11(3):193-203.

19. Van Houtven $\mathrm{CH}$, Ramsey SD, Hornbrook MC, Atienza AA, van Ryn M. Economic burden for informal caregivers of lung and colorectal cancer patients. Oncologist. 2010;15(8):883-893.

20. Li C, Zeliadt SB, Hall IJ, Smith JL, Ekwueme DU, Moinpour CM, et al. Burden among partner caregivers of patients diagnosed with localized prostate cancer within 1 year after diagnosis: an economic perspective. Support Care Cancer. 2013;21(12):3461-3469.

21. Hanly P, Ceilleachair AO, Skally M, O'Leary E, Kapur K, Fitzpatrick P, et al. How much does it cost to care for survivors of colorectal cancer? Caregiver's time, travel and outof-pocket costs. Support Care Cancer. 2013;21(9):2583-2592.

22. Mor V, Allen SM., Siegel K, Houts P. Determinants of need and unmet need among cancer patients residing at home. Health Serv Res. 1992;27(3):337-60.

23. Bishop MM, Beaumont JL, Hahn EA, Cella D, Andrykowski MA, Brady MJ et al. Late effects of cancer and hematopoietic stem-cell transplantation on spouses or partners compared with survivors and survivor-matched controls. J Clin Oncol 2007;25(11):1403-1411

24. Ferlay J, Soerjomataram I, Ervik M, Dikshit R, Eser S, Mathers C, et al. GLOBOCAN 2012 v1.0, Cancer Incidence and Mortality Worldwide. Lyon: IARC; 2012. Disonible en: http://globocan.iarc.fr/Pages/online.aspx

25. Rojas Tejada AJ, Fernández Prados JS, Pérez Meléndez C, editors. Investigar mediante encuestas: fundamentos teóricos y aspectos prácticos. Madrid: Síntesis; 1998.

26. Greene WH. Econometric Analysis. $3^{\circ} \mathrm{ed}$. New York: Prentice-Hall; 1997.

27. Francisco del Rey C, Mazarrasa Alvear L. Cuidados informales. Rev Enferm. 1995;18(202):61-65.

28. Conover WJ. Practical Nonparametric Statistics. $3^{\mathrm{a}}$ ed. New York, NY USA. Wiley; 1999.

29. Mohty B, Mohty M. Long-term complications and side effects after allogeneic hematopoietic stem cell transplantation: an update. Blood Cancer J. 2011;1(4):e16.

30. Goldzweig G, Rottenberg Y, Peretz T, Baider L. Silent Partners to Cancer Patients: Formal Caregivers and Oncologists. J Canc Edu. J Cancer Educ. 2014 Oct 16. [Epub ahead of print]. 
31. Jiménez-Martín S, Vilaplana Prieto C. The tradeoff between formal and informal care in Spain. Eur $\mathrm{J}$ Health Econ. 2012;13(4):461-490.

32. Vilaplana Prieto C, Jiménez-Martín S, García Gómez P. Trade-off entre cuidados formales e informales en Europa. Gac Sanit. 2011;25(S):115-124.

33. Del Pozo Rubio R, Escribano Sotos F. Impacto económico del cuidado informal tras la Ley de Promoción de la Autonomía Personal y Atención a las Personas en situación de Dependencia. Rev Esp Salud Pública. 2012;86(4):381-392.

34. Carreras E, Rovira M, Martinez C. Manual de trasplante hematopoyético. $4^{\mathrm{a}}$ ed. Barcelona: Editorial Antares; 2010.

35. Kristinsson SY, Landgren O, Dickman PW, Derolf AR, Bjorkholm M. Patterns of survival in multiple myeloma: a population-based study of patients diagnosed in Sweden from 1973 to 2003. J Clin Oncol. 2007;25(15):1993-1999.

36. Boletín Oficial del Estado. Ley 39/2006, de 14 de diciembre, de Promoción de la Autonomía Personal y Atención a las Personas en situación de Dependencia. BOE núm 299 de 15/12/2006.

37. Boletín Oficial del Estado. Resolución 10984/2010, de 29 de junio, sobre modificación del baremo de valoración de la situación de dependencia establecido en el Real Decreto 504/2007. BOE núm 168 de 12/07/2010. 